\title{
Clinical trends and outcomes of male breast cancer: Experience of a tertiary oncology centre in India
}

\author{
Anindya Mukherjee ${ }^{1}$, Aramita Saha ${ }^{2}$, Subrata Chattopadhyay', Prabir Kumar Sur ${ }^{3}$ \\ ${ }^{I}$ Department of Radiation Oncology, Medical College, Kolkata, India. \\ ${ }^{2}$ Department of Medical Oncology, Apollo Gleneagles Cancer Hospital, India. \\ ${ }^{3}$ Department of Radiotherapy, Hemalata Cancer Institute, Bhubaneswar, India.
}

Received March 06, 2014; Revised April 27, 2014; Accepted April 27, 2014; Published Online May 04, 2014

\section{Original Article}

\begin{abstract}
Purpose: Because of its rarity in any oncology centre, the clinical trends of male breast cancer specific to its geographical distribution have remained relatively unexplored. This study was done to analyze the clinico-pathological data, treatment given and survival patterns of male breast cancer patients visiting our tertiary medical centre and compare our results with available literature. Methods: All male breast cancer patients registered at our clinic from 2003 to 2009 were included. Frequency distribution analysis of the demographic and clinico-pathological data and treatment variables was done. Treatment outcome was examined from Kaplan-Meir survival estimates. Results: Thirty-three male breast cancer patients were encountered. The median age of presentation was sixty years. Mostly (87.9\%) they presented with lump in breast or axilla and were clinically staged to be ' 3 ' (57.6\%).Obesity and alcohol were the commonest risk factors identified. Modified radical mastectomy was the commonest (69.6\%) definitive therapy rendered with (only for clinically staged 3 patients) or without neo-adjuvant chemotherapy. Infiltrating ductal carcinoma was identified in most cases. Twenty-two patients received adjuvant chemotherapy and twenty-four received adjuvant radiotherapy. Eighteen (54.5\%) patients were hormone-receptor positive and received tamoxifen. The median Overall survival (OS) and Progression-free survival (PFS) came out to be 14.3 months (standard error, SE of 1.185; 95\% confidence interval, CI 12-16.6) and 15.7 (SE 5.35, 95\% CI 5.2-26.19) months respectively. Conclusion: Male breast cancers usually carry a poor prognosis due to presentation at later stages. Most of our results correlate with previous literature. Multi-centric prospective studies are required to validate the etiological factors and prognostic determinants of survival.
\end{abstract}

Keywords: Male Breast Cancer; Rare; Advanced Stage; Poor Outcome

\section{Introduction}

Male Breast Cancer (MBC) is a relatively rare entity ${ }^{1}$ accounting for less than $1 \%$ of all breast cancers and $1 \%$ of all malignancies in men. ${ }^{2}$ The Cancer Statistics, $2013{ }^{3}$ gives an estimate of 2240 new MBC cases to be diagnosed and 410 deaths to occur from the same by 2013 in US population. The mean age at diagnosis is 67 years (between 60 to 70 years) which is 5 years older than that in women ${ }^{1}$ in US, but in other parts of the world such as the Middle East and South

Corresponding author: Anindya Mukherjee; Department of Radiation Oncology, Medical College, Kolkata, India.

Cite this article as:

Mukherjee A, Saha A, Chattopadhyay S, Sur P. Clinical trends and outcomes of Male Breast Cancer: experience of a tertiary oncology centre in India. Int J Cancer Ther Oncol 2014; 2(3):02035.

DOI: $10.14319 /$ ijcto.0203.5
Asia, the age gap is smaller. ${ }^{4,5}$ As in women, breast cancer in males is also on the rise, the incidence has climbed $26 \%$ over the past 25 years. ${ }^{1}$ The incidence of MBC in Northwest Europe and North America is approximately 1/100,000. ${ }^{6}$

Genetic contributors to the risk of male breast cancer are similar, but not identical to those in women. Family history is relevant for both sexes ${ }^{7,8}$, and BRCA2 mutations and rearrangements play a particularly prominent role in male breast cancer.9,10,11 Conditions that alter the ratio of estrogen to androgen have been linked to breast cancer risk in men. Klinefelter's syndrome, exogenous estrogen or testosterone use, obesity and a history of prostate cancer treated with estrogens have been implicated. ${ }^{12-16}$ 
Tumor biology and clinic behaviour of MBC have been reported to closely correlate with breast cancers in women. Majority of MBCs are of infiltrating ductal histology ${ }^{12}$ and are estrogen receptor positive. ${ }^{17}$ Most commonly they present with lump in breast ${ }^{18,19}$ with positive axillary nodes. ${ }^{20,21}$ As in women, MBC survivorship is highly dependent on grade of tumors with grade 3 fairing much poorly than lower grades. ${ }^{21}$ Modified radical mastectomy has been recommended to be the standard of care. Post mastectomy Radiotherapy (PMRT) has been reported to prolong PFS ${ }^{22}$ and Local Recurrence free survival ${ }^{23}$, while systemic therapy has resulted in better outcomes in the node-positive patients. ${ }^{24}$ Tamoxifen therapy has been associated with improved recurrence free survival. ${ }^{24}$

In this retrospective study we have tried to analyze the demographic pattern and clinico-pathological characteristics of $\mathrm{MBC}$ in the Indian scenario. Considering the inter-play of prognostic determinants, how far the standard recommendations for management of MBC optimally affect survival in the Indian context has been our special point of interest.

\section{Methods and Materials}

A thorough search of the patients' registration records from December 2003 to December 2009 was undertaken to identify the male breast cancer patients. Data on patient history, relevant risk factors, presenting symptoms and signs, tumor pathology, clinical and/or pathological stage, treatment offered, details on recurrences were obtained from the patient case records.

TNM staging was done according to the $6^{\text {th }}$ American Joint Committee on Cancer (AJCC) classification. Diagnosis was done by fine needle aspiration cytology (FNAC) and/or from biopsy specimen. Mammography was not done in our study. Metastatic work-up included chest X-ray, ultrasonography of abdomen and bone scan and was done in almost all cases before definitive or adjuvant therapy was administered. Hormone receptor assay was done by immuno-histochemistry (IHC). Neo-adjuvant or adjuvant chemotherapy with appropriate pre-medications was administered and radiotherapy was given by Cobalt-60 machine. Tamoxifen was prescribed to hormone receptor positive patients in a dose of $20 \mathrm{mg}$ per oral daily for five years after adjuvant chemotherapy was completed.

After treatment completion, the patients were followed-up at three monthly basis till death or December 2012. Thorough clinical examination and imaging as and when required were done at each visit. Relevant data on time of occurrence and location of recurrences during this period were noted.

Frequency distribution tables and Kaplan - Meir estimates of survival were analysed by SPSS statistics software Version 20.0 compatible with Windows 7 edition.

\section{Results}

Thirty-three male breast cancer patients were treated in our institution from December 2003 to December 2009. The median age at diagnosis was sixty years (range, 45-71 years).

The clinical presentations were most commonly self-detected lump in the breast (twenty-nine patients, $87.9 \%$ ) or axilla (fifteen patients, $45.5 \%$ ), with or without pain. Nipple retraction and skin ulceration were found in $33.3 \%$ and $12.1 \%$ of the patients respectively. The lump was most commonly detected in the central quadrant $(39.3 \%)$ and the right breast (63.6\%) was more affected than the left. There were two patients who had undergone mastectomy earlier without any other treatment and presented with chest wall recurrence.

The risk factors commonly identified were obesity, high alcohol consumption in last three decades and gynaecomastia. The distribution of clinical presentations and risk factors among the patients are shown in Table 1.

The patients most commonly presented in clinical stage 3 (57.6\%).Tissue biopsy ( trucut or operative specimen) revealed modified Bloom Richardon score 3 in the majority (60.6\%); ER/PR hormone receptor positivity was found in $54.5 \%$ patients while seven patients had triple negative status. Almost all the cases were histopathologically Infiltrative Ductal Carcinoma. The pathological stage 3 was commonly (48.5\%) observed and lymph node positivity in surgical specimen was mostly between 1 - 3 nodes (30.3\%). Table 2 enlists these observations.

Primary treatment consisted of surgery. Modified radical mastectomy was done most commonly (69.6\%), two patients who presented with chest wall recurrence underwent toilet mastectomy. Four patients with metastatic presentation received no surgery. As per our institutional protocol, 'clinically staged 3' patients received neo-adjuvant chemotherapy (NACT) with either FAC (5-Fluorouracil, Adriamycin and Cyclophosphamide) or TAC (Docetaxel, Adriamycin and Cyclophosphamide) regimes. Clinically 'staged 1 or 2' patients $(30.3 \%)$ were operated upfront. The median duration for NACT was 69 days (standard deviation, SD-36 days) for two to three cycles. Patients with clinical stage 4 disease received upfront palliative chemotherapy with FAC regime (two patients) or Docetaxel alone (two patients), for a mean duration of 96 days (range 71-129 days). Two patients did not receive any form of therapy due to poor performance status $(\mathrm{ECOG} \geq 3)$. 
TABLE 1: Clinical presentation and Risk factors.

\begin{tabular}{lcc}
\hline \hline Clinical presentation & No. of patients (out of 33) & $\%$ \\
\hline Self detected lump in breast & 29 & 87.9 \\
Self-detected lump in axilla & 15 & 45.5 \\
Pain & 25 & 45.5 \\
Nipple retraction & 11 & 33.3 \\
Skin ulceration & 4 & 12.6 \\
Mastectomy done earlier & 2 & 6.0 \\
Laterality: & & \\
\hline Right & 21 & 63.6 \\
Left & 12 & 36.3 \\
Location: & & \\
\hline Upper outer quadrant & 7 & 21.2 \\
Upper inner quadrant & 3 & 9.0 \\
Lower outer quadrant & 6 & 18.1 \\
Lower inner quadrant & 4 & 12.1 \\
Central quadrant & 13 & 39.3 \\
Risk Factors: & & \\
\hline Obesity & 14 & 42.4 \\
Heavy alcohol consumption & 15 & 45.5 \\
Gynaecomastia & 2 & 6 \\
Family history & 2 & 6 \\
Previous radiation exposure & 1 & 3 \\
Klinefelter's syndrome & 0 & 0 \\
Occupational exposure & 2 & 6 \\
Prostate cancer & 4 & 12.1 \\
\hline \hline
\end{tabular}

TABLE 2: Clinical Stage and Histopathology

\begin{tabular}{|c|c|c|}
\hline Clinical Stage & No. of patients(out of 33) & $\%$ \\
\hline 1 & 2 & 6.1 \\
\hline 2 & 8 & 24.2 \\
\hline 3 & 19 & 57.6 \\
\hline 4 & 4 & 12.1 \\
\hline \multicolumn{3}{|l|}{ HISTOPATHOLOGY } \\
\hline \multicolumn{3}{|l|}{ Bloom Richardson grade : } \\
\hline 1 & 2 & 6.1 \\
\hline 2 & 8 & 24.2 \\
\hline 3 & 20 & 60.6 \\
\hline Missing & 3 & 9.1 \\
\hline \multicolumn{3}{|c|}{ Hormone receptor positivity: } \\
\hline ER/ PR +, Her2 neu - & 18 & 54.5 \\
\hline ER/ PR -, Her2 neu + & 3 & 9 \\
\hline ER/ PR - ,Her2 neu - & 7 & 21.2 \\
\hline ER/PR+ ,Her2 neu + & 0 & 0 \\
\hline Receptor status unknown & 5 & 15.2 \\
\hline \multicolumn{3}{|l|}{ Histologic type: } \\
\hline IDC & 31 & 93.9 \\
\hline Padget's disease & 2 & 6 \\
\hline Others & 0 & 0 \\
\hline
\end{tabular}

In the adjuvant setting, only the two patients who underwent toilet mastectomy received docetaxel; rest (66.6\%) were treated with FAC regime. The mean duration for adjuvant chemotherapy was 70 days (SD-2.2 days) for those who received NACT and 127 days (SD-2.2 days) when no NACT was administered. Twenty-four (72.7\%) patients received adjuvant radiotherapy to a dose of 50 Gray in 25 fractions (conventional fractionation) by Cobalt-60 machine. Hormone therapy consisted of tamoxifen alone and was given to in receptor positive status (54.5\%). Salvage orchiectomy was done in two patients. The above described treatment variables, lymph node harvest and pathological stage are summarized in Table 3. 
TABLE 3: Treatment Variables and Pathological Staging

\begin{tabular}{|c|c|c|}
\hline "TREATMENT VARIABLES & No. of patients (out of 33) & $\%$ \\
\hline Modified Radical mastectomy & 23 & 69.6 \\
\hline Radical mastectomy & 2 & 6 \\
\hline Toilet mastectomy & 2 & 6 \\
\hline Orchiectomy & 2 & 6 \\
\hline NACT-FAC regime & 13 & 39.4 \\
\hline NACT-TAC regime & 4 & 12.1 \\
\hline Upfront Palliative CT(FAC regime) & 2 & 6 \\
\hline Upfront palliative CT(Docetaxel) & 2 & 6 \\
\hline Adjuvant CT-FAC regime & 22 & 66.6 \\
\hline Adjuvant CT- Docetaxel & 2 & 6 \\
\hline Adjuvant RT & 24 & 72.7 \\
\hline No treatment received & 2 & 6 \\
\hline \multicolumn{3}{|l|}{ Pathological lymph nodes positive: } \\
\hline Nil & 3 & 9.1 \\
\hline $1-3$ & 10 & 30.3 \\
\hline $4-10$ & 6 & 18.2 \\
\hline$>10$ & 6 & 18.2 \\
\hline Missing & 8 & 24.2 \\
\hline \multicolumn{3}{|l|}{ Pathological stage: } \\
\hline 1 & 1 & 3 \\
\hline 2 & 8 & 24.2 \\
\hline 3 & 16 & 48.5 \\
\hline 4 & 4 & 12.1 \\
\hline Missing & 4 & 12.1 \\
\hline
\end{tabular}

TABLE 4: Pathological Stage Specific Over-all Survival (OS) and Progression-Free Survival (PFS):

\begin{tabular}{ccccccc}
\hline \hline & \multicolumn{3}{c}{$\begin{array}{c}\text { OS } \\
\text { (months) }\end{array}$} & \multicolumn{2}{c}{$\begin{array}{c}\text { PFS } \\
\text { (months) }\end{array}$} \\
\hline Stage & Median OS & Std. error & Confidence interval & Median PFS & Std error & Confidence Interval \\
\hdashline 1 & $36.2^{*}$ & & & & $36.2^{*}$ \\
2 & 32.9 & 7.77 & $17.85-48.14$ & 30.53 & 3.82 & $23.03-38.02$ \\
3 & 14.2 & 1.242 & $11.76-16.63$ & 11.2 & 0.687 & $9.85-12.54$ \\
4 & 5.46 & 0.12 & $5.22-5.69$ & $5.46^{*}$ & $0.12 *$ & $5.22-5.69^{*}$ \\
\hline \hline
\end{tabular}

${ }^{*}=$ Stage wise median progression free survival

The median follow-up period was seven months (SD -10.4 months), ranging from zero to thirty-six months. The recurrences were mostly distal-lung (8 patients, $24.2 \%)$ followed by liver ( 5 patients, $15.1 \%$ ), bone ( 2 patients, $6 \%$ ) and brain (1 patient). Local recurrence was observed in $2(6 \%)$ patients. Most (12 patients, $61.1 \%$ ) of the recurrences occurred in the first year of follow-up. The median over-all survival (OS) and Progression-free survival (PFS) came out to be 14.3 months (standard error, SE of 1.185; 95\% confidence interval, CI 12-16.6) and 15.7 months (SE 5.35, 95\% CI 5.2-26.19) respectively. The stage specific OS and PFS are shown in Table 4 and graphically represented by Kaplan -Meir curves in Figure 1 and 2.There were significant differences in OS $(\log$ rank test $=0.00)$ and PFS (log rank test $=0.02)$ between different pathological stages.

\section{Discussion}

Our study corroborates with the theory that MBC has a peak incidence 5-10 years later than that in females, since the highest risk of breast cancers in females in India is between 45-50 years. The concept of smaller age gap in South Asian scenario ${ }^{4,5}$, is however not supported by this study.

15-20\% MBC patients have a positive family history of breast cancer in a first degree relative. ${ }^{7,8} \mathrm{We}$ found two patients (6\%) who had sisters with breast cancers. However the study by Hill et al ${ }^{25}$ on family history in MBC showed that it was not associated with mean age at onset for the men nor was it associated with survival. An abnormal oestrogen to androgen ratio is also a known risk factor for the disease. Klinefelter's syndrome ${ }^{13}$, exogenous oestrogen ${ }^{14}$, obesity ${ }^{15,16}$ and a history of prostate cancer ${ }^{26}$ are all associated with this hormonal imbalance. Also, prior exposure to radiation or electro-magnetic fields ${ }^{27}$ and occupational exposure to volatile aromatic hydrocarbons ${ }^{28}$ contribute to the risk. This study agrees with this view, with special emphasis on obesity and heavy alcohol consumption ${ }^{29}$ as the commonest risk factors found in more than $40 \%$ of our patients. Risk estimates of the association between gynecomastia and MBC are unstable 
due to the small sample sizes available. A recent European case-control study of $74 \mathrm{MBC}$ cases and 1,432 population-based controls ${ }^{29}$ observed a significant elevation in risk for $\mathrm{MBC}$ associated with gynecomastia. We had two patients $(6 \%)$ with gynaecomastia in this study.

Inherited mutations and rearrangements of BRCA gene increase the risk of MBC, more so with BRCA 2 (5-15\%) than BRCA 1 (less than 5\%). ${ }^{8,10}$ Data are mixed regarding the relevance of other germ-line mutations such as those in PALB2, the androgen receptor (AR), CYP17, and CHEK2. ${ }^{30-32} \mathrm{Ge}-$ netic testing for any of these mutations was not done in any of our patients.

Consistent with previous literature ${ }^{18,19}$, the most common presentation was a painless sub-areolar lump detected in $87.9 \%$ of our patients. The most common location in women is the upper outer quadrant. This gender differences is likely due to the paucity of breast tissue in males compared to the females. ${ }^{1}$ Nipple involvement and lymph node positivity ${ }^{20,21}$ has been reported to be more common with MBC than female breast cancers. In our study, eleven (33.3\%) patients presented with nipple retraction and fifteen (45.5\%) with axillary lump.

The staging of MBCs follows the same TNM classification as in women. The high propensity of lymph node metastasis has been attributed to the later stages of presentation of
MBCs compared to the female counterparts. More than 50\% of MBCs are diagnosed at stage 3 and beyond. ${ }^{1}$ Consistent with this observation, our patients most commonly $(57.6 \%)$ presented in stage 3 . Breast conservation surgery is usually not advocated in MBCs due to the inadequacy of breast tissue and MRM has been recognised to be the standard procedure. ${ }^{22,33}$ Twenty-nine (69.6\%) of the patients of our study underwent MRM as the curative surgery.

Majority of MBCs have infiltrating ductal type histology. ${ }^{26}$ Other histologies like ductal carcinoma in situ(DCIS), lobular carcinoma in situ(LCIS), mixed ductal and cribriform, Paget's disease, inflammatory, etc have been reported in literature. Almost all (93.9\%) of our patients had infiltrating ductal histopathology. Consistent with the reports of MBCs being usually hormone receptor positive ${ }^{17}$, eighteen patients (54.5\%) in this study were found to have both estrogen and progesterone receptors(ER, PR) by IHC. Recent studies 34 have shown lower rates of Her2neu positivity (2-15\%) in men than in women (18-20\%). Her2neu positivity was found in three patients and triple negative status in seven patients of our study. Lymph nodal involvement is tightly linked to breast cancer outcomes in men. ${ }^{16,35-37}$ Lymph nodal harvest between one to three was commonly (30.3\%) observed in our study. Smaller studies ${ }^{38}$ have demonstrated the feasibility of sentinel node biopsy, but it is yet to receive standard recommendation. So it was not performed in any of our patients.

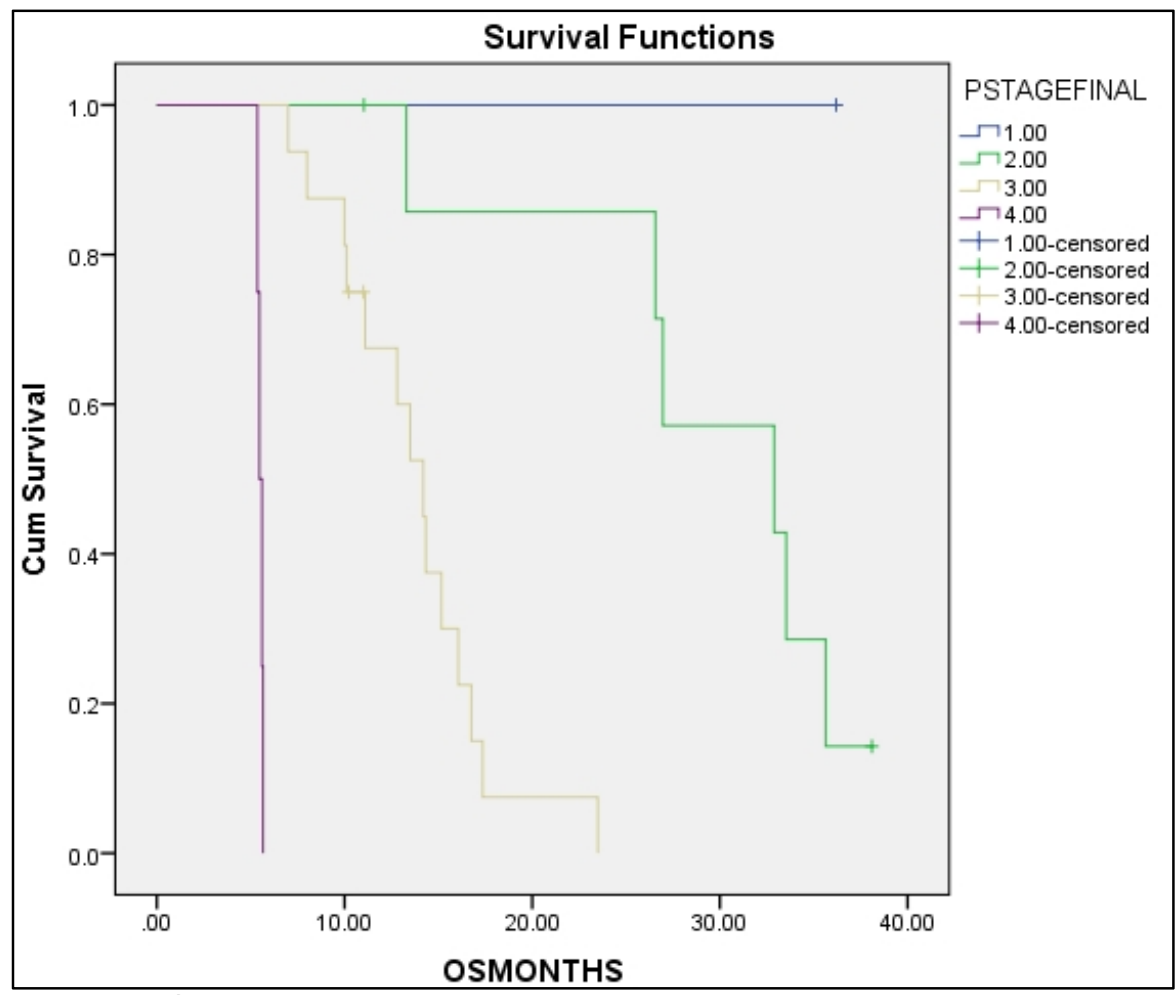

FIG. 1: Pathological Stage Specific Kaplan-Meir estimates of over-all survival.

Test of equality of survival distributions for the different levels of pathological stage by Log-rank test $=0.00$ (significant) 


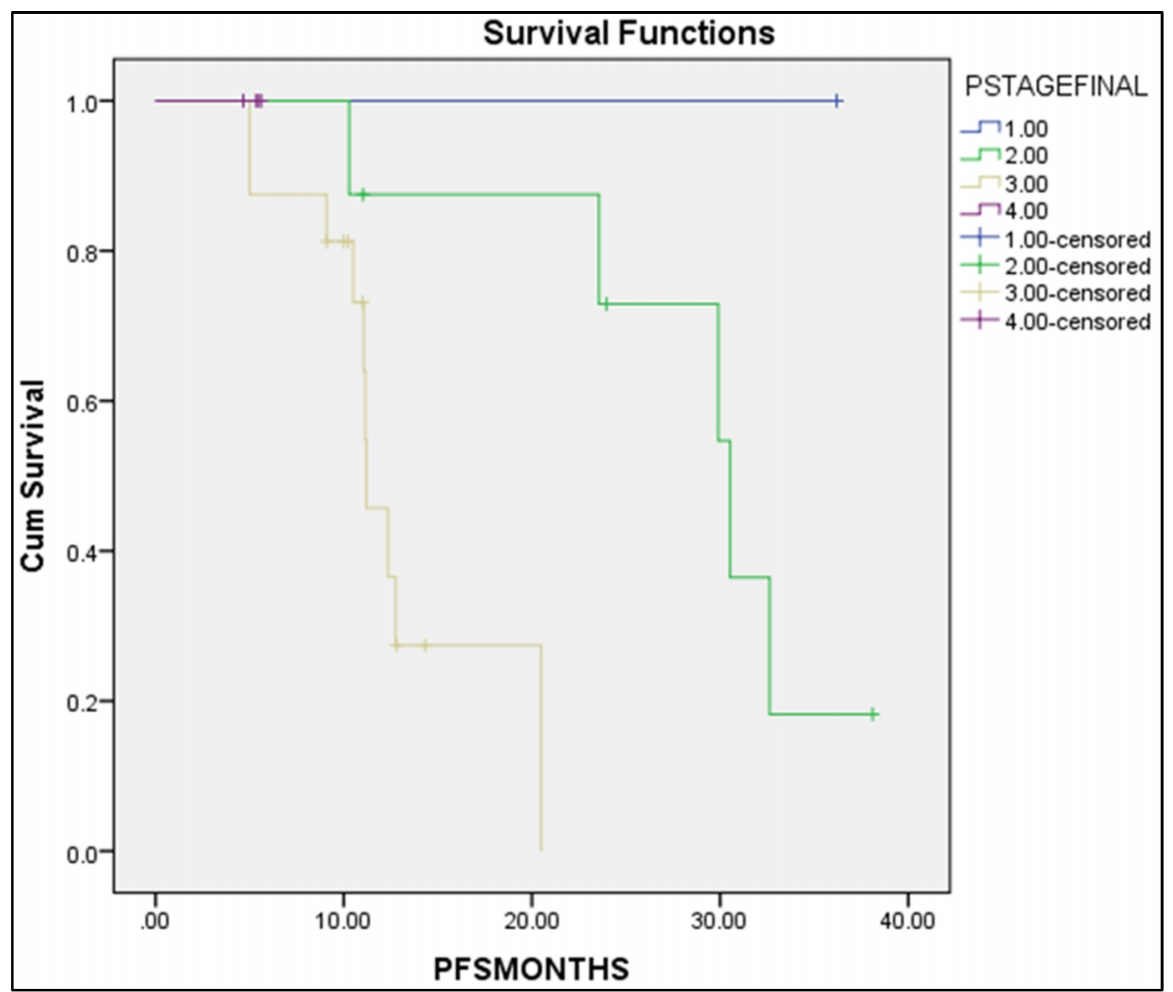

FIG. 2: Pathological Stage Specific Kaplan-Meir estimates of Progression-Free Survival

Test of equality of survival distributions for the different levels of pathological stage by Log-rank test $=0.02$ (significant).

With a specificity of $90 \%{ }^{22}$ mammography can aptly differentiate between gynaecomastia and MBC. ${ }^{39}$ However the rarity of this disease and the relative ease of diagnosis by physical examination don't support routine mammographic screening. Also, most of the patients presented with palpable tumors at presentation. For these two reasons, mammography was not used in our study.

Neo-adjuvant chemotherapy is not yet the standard protocol for locally advanced MBCs. However, we have used it for clinical stage 3 patients as an extrapolation of data from female breast cancers as per our institutional protocol. Data on adjuvant systemic chemotherapy are limited and whether it significantly impacts survival is yet to be validated. However, it has been associated with better outcomes in node positive patients. ${ }^{24,40}$ Two regimes- FAC and single agent docetaxel have been used in this study. The choice of neo-adjuvant (FAC or TAC regime) or adjuvant chemotherapy was not validated and impact on survival not analyzed. The impact of PMRT on PFS and L-RFS ${ }^{23}$ prompted us to use it to a total dose of 50 Gray in conventional fractionation over five weeks, following the same indications followed in female breast cancers. However, fewer numbers of patients in previous studies ${ }^{23}$ limits the substantiality of PMRT translating to a survival benefit. Tamoxifen has been found to prolong RFS ${ }^{24}$ and it is the accepted standard of care for receptor positive $\mathrm{MBC}$. Hence, it was given to eighteen (54.5\%) of the patients with ER, PR positive status. Treat- ment for metastatic disease in men has been primarily evaluated in case reports and small case series. In a Spanish series of fifty men with breast cancer, ten of whom were treated with endocrine therapy for metastatic disease (either orchiectomy, estrogen, or tamoxifen), two of the ten had complete responses, one of which lasted sixty months. ${ }^{41}$ Data regarding use of aromatase inhibitors in conjunction with gonadotropin releasing hormone agonist or orchiectomy to achieve complete oestrogenic suppression is far too limited to be accepted as a recommendation. None of the patients received aromatase inhibitors. In our study palliative chemotherapy alone was offered to metastatic cases, upfront or after recurrences.

Survival in MBCs has been chiefly reported based on grade and stage of the disease. In forty-three Canadian patients ${ }^{21}$, 5 -year survival was $58 \%$ in those with grade 2 tumors and $45 \%$ in those with grade 3 tumors. Several studies 1,42, 43, 44 have consistently reported stage-specific 5-year OS of more than $80 \%$ in stage $1,50-80 \%$ in stage $2,20-50 \%$ in stage 3 and $10 \%$ or less in stage $4 \mathrm{MBCs}$. As compared to females, equivalent OS 1,45 has been observed in age and stage-matched patients, while disease specific survival was significantly better in males ${ }^{46}$ than in females. In our study, at the end of 3 year follow-up, the median OS came out to be 36.9 months, 32.9 months, 14.2 months and 5.46 months for pathological stages $1,2,3$ and 4 respectively. Similar trend was reported in progression-free survival also. Thus, the 
prognostic impact of stage was consistent with reported trends of survival.

The analysis of the patterns of recurrences clearly shows that distal recurrences fairly outnumber the local recurrences. The most common site of distal recurrence was lung (24.2\%) followed by liver (15.1\%). This proves the high propensity of haematogeneous metastasis of MBC irrespective of the stage of disease.

This study is representative of the scenario in eastern India. The limitations of this study include small sample size, retrospective data and single-institute experience. Also genetic testing to detect genetic preponderances could not be done due to financial inadequacy. Whether our institutional protocol for NACT in MBC positively impacts the outcome needs to be validated by further studies. In short, we have shown here that in our centre, the course of this disease and outcome agrees mostly with other centres. However, the search for newer treatment protocols to reduce the disease burden and mortality still remains unanswered.

\section{Conclusion}

Although MBC has quite a number of similarities with female breast cancer, it can never be considered analogous to the latter. The rarity of this disease delays the diagnosis and patients usually present at later stages at our centre. This confers a worse prognosis to all MBC patients in general. Screening of patients with gynaecomastia for BRCA 2 gene mutations may be contemplated if financial resources are supportive. To conclude, in order to address the demographic variations of $\mathrm{MBC}$ and validate the impact of each prognostic determinant, a bigger pooled data from a multi-institutional prospective study need to be analyzed.

\section{Conflict of interest}

The authors declare that they have no conflicts of interest. The authors alone are responsible for the content and writing of the paper.

\section{References}

1. Giordano SH, Cohen DS, Buzdar AU, et al. Breast carcinoma in men: a population-based study. Cancer 2004; 101:51-7.

2. Fentiman IS, Fourquet A, Hortobagyi GN. Male breast cancer. Lancet 2006; 367:595-604.

3. Siegel R, Naishadham D, Jemal A. Cancer Statistics, 2013. CA Cancer J Clin 2013; 63:11-30.

4. Salehi A, Zeraati H, Mohammad K et al. Survival of male breast cancer in Fars,South of Iran. Iran Red Crescent Med J2011; 13:99-105.
5. Tawil AN, Boulos FI, Chakhachiro ZI et al. Clinicopathologic and immunohistochemical characteristics of male breast cancer: a single center experience. Breast J2012; 18: 65-8.

6. Hodgson NC, Button JH, Franceschi D, et al. Male breast cancer:is the incidence increasing? Ann Surg Oncol 2004; 11:751-5.

7. Brinton LA, Richesson DA, Gierach GL, et al. Prospective evaluation of risk factors for male breast cancer. I Natl Cancer Inst 2008; 100: 1477-81.

8. D’Avanzo B, La Vecchia C. Risk factors for male breast cancer. Br J Cancer 1995; 71: 1359-62.

9. Gilbert SF, Soliman AS, Karkouri M, et al. Clinical profile, BRCA2 expression, and the androgen receptor CAG repeat region in Egyptian and Moroccan male breast cancer patients. Breast Dis 2011; 33: 17-26.

10. Ottini L, Rizzolo P, Zanna I, et al. BRCA1/BRCA2 mutation status and clinicalpathologic features of 108 male breast cancer cases from Tuscany: a population-based study in central Italy. Breast Cancer Res Treat 2009; 116: 577-86.

11. Kwiatkowska E, Teresiak M, Breborowicz D, et al. Somatic mutations in the BRCA2 gene and high frequency of allelic loss of BRCA2 in sporadic male breast cancer. Int J Cancer 2002; 98:943-5.

12. Hultborn R, Hanson C, Kopf I, et al. Prevalence of Klinefelter's syndrome in male breast cancer patients. Anticancer Res 1997; 17: 4293-7.

13. Medras M, Filus A, Jozkow P, et al. Breast cancer and long-term hormonal treatment of male hypogonadism. Breast Cancer Res Treat 2006; 96: 263-5.

14. Brinton LA, Carreon JD, Gierach GL, et al. Etiologic factors for male breast cancer in the U.S. Veterans Affairs medical care system database. Breast Cancer Res Treat 2010; 119: 185-92.

15. Altinli E, Gorgun E, Karabicak I, et al. Anthropometric measurements in male breast cancer. Obes Surg 2002; 12: 869-70.

16. Thellenberg C, Malmer B, Tavelin B, et al. Second primary cancers in men with prostate cancer: an increased risk of male breast cancer. J Urol 2003; 169:1345-8.

17. Anderson WF, Jatoi I, Tse J, et al. Male breast cancer: a population-based comparison with female breast cancer. J Clin Oncol 2010; 28: 232-9.

18. Sandler B, Carman C, Perry RR. Cancer of the male breast. Am Surg 1994; 60: 816-20.

19. Shukla NK, Seenu V, Goel AK, et al. Male breast cancer: a retrospective study from a regional cancer center in northern India. J Surg Oncol 1996; 612:143-148.

20. Joshi MG, Lee AK, Loda M, et al. Male breast carcinoma: an evaluation of prognostic factors con- 
tributing to a poorer outcome. Cancer 1996; 77: 490-498.

21. McLachlan SA, Erlichman C, Liu FF, et al. Male breast cancer: an 11 year review of 66 patients. Breast Cancer Res Treat 1996; 40: 225-30.

22. Schaub NP, Maloney N, Schneider $\mathrm{H}$, et al. Changes in male breast cancer over a 30-year period. Am Surg 2008; 74: 707-11.

23. Yu E, Suzuki H, Younus J, et al. The impact of post-mastectomy radiation therapy on male breast cancer patients - a case series. Int J Radiat Oncol Biol Phys 2012; 82: 696-700.

24. Giordano SH, Perkins GH, Broglio K, et al. Adjuvant systemic therapy for male breast carcinoma. Cancer 2005; 104: 2359-64.

25. Hill A, Yagmur Y, Tran KN, et al. Localized male breast carcinoma and family history. An analysis of 142 patients. Cancer 1999; 86:821-5.

26. Amir H, Hirji KF. Carcinoma of the male breast in Tanzania. J Natl Med Assoc 1992; 84: 337-40.

27. Matanoski GM, Breysse PN, Elliott EA. Electromagnetic field exposure and male breast cancer. Lancet 1991; 337: 737.

28. Hansen J. Elevated risk for male breast cancer after occupational exposure to gasoline and vehicular combustion products. Am J Ind Med 2000; 37:349-52.

29. Guenel P, Cyr D, Sabroe S, et al. Alcohol drinking may increase risk of breast cancer in men: a European population-based case-control study. Cancer Causes Control 2004; 15: 571-80.

30. Wasielewski M, den Bakker MA, van den Ouweland A, et al. CHEK2 1100delC and male breast cancer in the Netherlands. Breast Cancer Res Treat 2009; 116: 397-400.

31. Ding YC, Steele L, Kuan CJ, et al. Mutations in BRCA2 and PALB2 in male breast cancer cases from the United States. Breast Cancer Res Treat 2011; 126: 771-8.

32. Young IE, Kurian KM, Annink C, et al. A polymorphism in the CYP17 gene is associated with male breast cancer. Br J Cancer 1999; 81: 141-3.

33. Zhou FF, Xia LP, Guo GF, et al. Changes in therapeutic strategies in Chinese male patients with breast cancer: 40 years of experience in a single institute. Breast 2010; 19: 450-5.

34. Rudlowski C, Friedrichs N, Faridi A, et al. Her-2/neu gene amplification and protein expres- sion in primary male breast cancer. Breast Cancer Res Treat 2004; 84:215-23.

35. Arslan UY, Oksuzoglu B, Ozdemir N, et al. Outcome of non-metastatic male breast cancer: 118 patients. Med Oncol 2012; 29: 554-60.

36. Cutuli B, Lacroze M, Dilhuydy JM, et al. Male breast cancer: results of the treatments and prognostic factors in 397 cases. Eur J Cancer 1995; 31A:1960-4.

37. Cutuli B, Le-Nir CC, Serin D, et al. Male breast cancer. Evolution of treatment and prognostic factors. Analysis of 489 cases. Crit Rev Oncol Hematol 2010; 73: 246-54.

38. Boughey JC, Bedrosian I, Meric-Bernstam F, et al. Comparative analysis of sentinel lymph node operation in male and female breast cancer patients. J Am Coll Surg 2006; 203: 475-80.

39. Evans GF, Anthony T, Turnage RH, et al. The diagnostic accuracy of mammography in the evaluation of male breast disease. Am J Surg 2001; 181:96-100.

40. Yildirim E, Berberoglu U. Male breast cancer: a 22-year experience. Eur J SurgOncol 1998; 24: 548-52.

41. Izquierdo MA, Alonso C, De Andres L, et al. Male breast cancer. Report of a series of 50 cases. Acta Oncol 1994; 33: 767-71.

42. de Perrot M, Deleaval J, Robert J, Spiliopoulos A. Thirty-year experience of surgery for breast carcinoma in men. Eur J Surg 2000; 166:929-31.

43. Vaizey C, Burke M, Lange M. Carcinoma of the male breast - a review of 91 patients from the Johannesburg Hospital breast clinics. S Afr J Surg 1999; 37:6-8.

44. Rudan I, Rudan N, Basić N, Basić V, Rudan D. Differences between male and female breast cancer. II. Clinicopathologic features. Acta Med Croatica 1997; 51:129-33.

45. Adami HO, Holmberg L, Malker B, Ries L. Long-term survival in 406 men with breast cancer. Br J Cancer 1985; 52:99-103.

46. Scott-Conner CE, Jochimsen PR, Menck HR, et al. An analysis of male and female breast cancer treatment and survival among demographically identical pairs of patients. Surgery 1999; 126: 775-80. 